\title{
Relativistic Coulomb excitation of the giant dipole resonance in nuclei: A straightforward approach
}

\author{
C. H. Dasso and M. I. Gallardo \\ Departamento de Física Atómica, Molecular y Nuclear Facultad de Física, Apartado 1065, 41080 Sevilla, Spain \\ H. M. Sofia \\ Departmento de Física, Comisión Nacional de Energía Atómica and CONICET, Av. del Libertador 8250, 1429 Buenos Aires, Argentina \\ A. Vitturi \\ Dipartimento di Fisica and INFN, Via Marzolo 8, I-35131 Padova, Italy \\ (Received 3 May 2004; published 11 October 2004)
}

\begin{abstract}
We investigate alternatives to the standard formalism used for the study of relativistic Coulomb excitation of the giant dipole resonance in nuclei. The idea is to obtain reasonable results for the probabilities of excitation and cross sections to the one-phonon and two-phonon levels avoiding the substantial complexity of the treatments exploited so far. This is achieved for the relevant range of partial waves up to bombarding energies of at least $5 \mathrm{GeV}$ per nucleon. The transfer of energy to the center of mass of the excited nuclei is also investigated.
\end{abstract}

DOI: 10.1103/PhysRevC.70.044903

PACS number(s): 25.70.De, 25.75.- $\mathrm{q}$

\section{INTRODUCTION}

Heavy ion Coulomb excitation has been traditionally analyzed using semiclassical time-dependent approximations. That is to say, with the combination of a classical treatment for the relative motion of the colliding nuclei with a quantum-mechanical description of the excitation of the intrinsic degrees of freedom of the nuclear system $[1,2]$.

In recent years the experimental activity has gradually shifted the interest towards intermediate and high-energy collisions, where the large relative velocities of the ions involved in the process make it necessary to implement a relativistic formulation of the problem. Several authors [3-15] have calculated probabilities for relativistic Coulomb excitation (RCE) following the ideas developed in a seminal contribution by Alder and Winther [16]. Their approach exploits a multipole expansion of the interacting potentials that is best formulated in terms of the Fourier transforms of the timedependence of the retarded electromagnetic couplings, where useful analytic expressions apply. We can refer to this as working in the " $\omega$ "-representation, in contrast to working in the more familiar time, or " $t$ "-representation.

The implementation of this scheme has confronted a number of serious numerical difficulties. This is particularly true when the problem requires a multistep description of the excitation process, the simple first-order approach being inadequate. Complications also arise when more elaborate microscopic descriptions of the nuclear structure lead to a large number of channels and/or introduce anharmonic corrections in the form factors connecting the different states. This is the reason why the original formulation of the relativistic Coulomb excitation problem has often resorted to various approximation schemes such as the long-wavelength approximation or first-order Born approximation.

Given the traditional simplicity of semiclassical approximations to nuclear reactions, however, it is almost inevitable to wonder if the implementation of such formidable formalism is indeed unavoidable. Let it be clear, from the beginning, that we do not intend to cast any doubt in the ultimate validity of the standard approach and the analyses of the RCE process that already exist in the literature. What we are talking about here is a genuine curiosity for learning about the extent to which the high price paid for adopting a rather complicate calculation scheme is actually justified for understanding the results obtained at the current range of experimental bombarding energies.

First of all one would like to test if the calculation in the $\omega$-representation is compelling, or one could-more transparently and without loss of accuracy - formulate the excitation problem in the usual time-representation. Also, it seems important to determine if the very specific details of the structural models that are used to represent the GDR are crucial to solve the problem or, in retrospective, rather unimportant in leading order.

In what follows we set to check out these premises. Behind our motivation is the realization that one normally makes use of the dipole approximation in the characterization of the electromagnetic exciting fields (derived from both the relativistic scalar and vector retarded potentials). As long as this is the case, the results of the calculation will be mostly determined by the displacement and current (read "velocity") associated with the movement of the center of charge. Thus, the familiar model in which one takes the oscillation of protons against neutrons in a pure vibrational motion may suffice.

Following this Introduction we present, in Sec. II, a calculation scheme that yields the probabilities of excitation of the GDR and the double giant dipole resonance (DGDR) from the solution of the entirely analogous classical problem by well-tested projection rules. Section III develops an alternative method to evaluate the same quantities but from a quantal, coupled-channel formulation. In Sec. IV we com- 
pare the RCE probabilities obtained by both these approaches with exact, state-of-the-art calculations according to the standard prescriptions of Alder and Winther [16]. The widespread practice of taking as a reference system the (accelerated!) center of mass of the target can be easily examined within our perspective and this is done in detail in Sec. $\mathrm{V}$. The aim is here to investigate if the presence of inertial forces can significantly alter the excitation probabilities constructed in Secs. II and III. A brief summary and some conclusions close the paper in Sec. VI. Two very compact computer programs, useful to calculate excitation probabilities of the GDR and DGDR up to energies of $5 \mathrm{GeV}$ per nucleon, are included in Appendices A and B.

\section{CLASSICAL EQUATIONS OF MOTION}

The simplest calculation we can contemplate is that of interpreting the dipole mode in terms of the excitation of a three-dimensional spring joining the centers of mass of the $Z_{T}$ protons and $N_{T}$ neutrons. From now on the indices $P$ and $T$ stand for "projectile" and "target," respectively. For the moment we neglect the motion of the target as a whole and let the origin of our coordinate system coincide with the total center of mass of the nucleus whose dipole mode we intend to investigate. This is not a priori justified and therefore we will come back to examine the validity of this ansatz later on.

We set to calculate the classical motion of this oscillator as determined by the electrical and magnetic forces that follow from the Lienard-Wiechert relativistic interaction. These potentials are generated by a projectile which moves in the direction $y$ at relativistic energies, i.e., with a velocity $v_{p}$ close to the speed of light $c$. The energy of this particle is so large compared with the characteristic energy $\hbar \omega$ associated with the excitation of the giant dipole mode of the target at the zero-, one-, and two-phonon levels that the approximation in which the trajectory of its motion is uniform and rectilinear is fully justified. The case we consider is that of an incident particle with an impact parameter $b$ and we take the reaction plane to coincide with the $(x, y)$-plane.

Making use of the dipole approximation we express, in a neighborhood of the origin, the relevant components of the electric and magnetic fields felt by the charge at one end of the spring as [17]

$$
\begin{gathered}
E_{x}(t)=\frac{Z_{P} e \gamma b}{\left(b^{2}+\gamma^{2} v_{P}^{2} t^{2}\right)^{3 / 2}}, \\
E_{y}(t)=\frac{Z_{P} e \gamma v_{P} t}{\left(b^{2}+\gamma^{2} v_{P}^{2} t^{2}\right)^{3 / 2}}, \\
B_{z}(t)=\frac{v_{P}}{c^{2}} E_{x}(t),
\end{gathered}
$$

where $\gamma=1 / \sqrt{1-\beta^{2}}$ and $\beta=v_{p} / c$. Components of the force in the $z$ direction are irrelevant as the motion is, under the assumption that the spring is initially not elongated and at rest, constrained to remain on the reaction plane.
Enforcing the condition that the center of mass of the target is at rest the equation of motion for the vector $\vec{r}(t)$ $\equiv(x(t), y(t), 0)$ that represents the separation distance between protons and neutrons as a function of time becomes

$$
D \frac{d^{2} \vec{r}(t)}{d t^{2}}=-C \vec{r}(t)+Z_{T} e \delta\left[\vec{E}(t)+\delta \frac{\vec{d} r}{d t} \times \vec{B}(t)\right],
$$

where

$$
D=\frac{N_{T} Z_{T}}{N_{T}+Z_{T}} m \text { and } \quad C=D \omega^{2}
$$

are, respectively, the mass and restoring force parameters corresponding to the effective one-body problem associated with the mode and $m$ the mass of a nucleon. The factor $\delta$ $=N_{T} /\left(N_{T}+Z_{T}\right)$ gives the scale between the actual displacement in space of the center of charge and the relative coordinate $\vec{r}$.

Once the effects of the driving forces in Eq. (2.2) cease to exist the harmonic mode reaches an asymptotic state with an excitation energy $E_{\infty}$. This value, in turn, can be converted into an average number of phonons $N_{\infty}=E_{\infty} / \hbar \omega$. Notice that as far as calculating $E_{\infty}$ is concerned one can simplify things further by ignoring the presence of the magnetic field term in Eq. (2.2). This is not so, of course, if one is interested in obtaining trajectories of motion in space (as it will be the case in Sec. IV).

Provided that $N_{\infty}$ is very small compared to one we can estimate in perturbation theory the probabilities for the excitation of the first three levels of the harmonic ladder as

$$
P_{0} \approx 1-N_{\infty}, P_{1} \approx N_{\infty}, P_{2} \approx\left(P_{1}^{2}\right) / 2
$$

Notice that normalization is conserved here up to the order of $P_{2}$, a number that should be even much smaller than $P_{1}$ with respect to one.

A computer code RCE has been written (see Appendix A) to construct the excitation probabilities according to this most simple scheme. We shall show results and compare with the standard formalism later on in Sec. IV.

\section{COUPLED-CHANNEL FORMALISM}

We work out in this section a quantal counterpart to the classical problem just described. We choose the same system of coordinates as before and express the relativistic Hamiltonian that describes the intrinsic excitation of the dipole mode as

$$
H=\frac{p_{x}^{2}+p_{y}^{2}+p_{z}^{2}}{2 D}+C \frac{x^{2}+y^{2}+z^{2}}{2}+V(\vec{r}, \vec{p}, t),
$$

where we have identified the same three-dimensional harmonic oscillator Hamiltonian $H_{0}$ as in Sec. II, now affected by a time-dependent perturbation 


$$
\begin{aligned}
V(\vec{r}, \vec{p}, t)= & Z_{T} e \Phi(\delta \vec{r})-\frac{Z_{T} e \delta}{2 D c}(\vec{p} \cdot \vec{A}(\delta \vec{r})+\vec{A}(\delta \vec{r}) \cdot \vec{p}) \\
& +\frac{Z_{T}^{2} e^{2} \delta}{2 D c^{2}} \vec{A}(\delta \vec{r}) \cdot \vec{A}(\delta \vec{r}) .
\end{aligned}
$$

In Eq. (3.2) the scalar and vector potentials are, respectively,

$$
\begin{gathered}
\Phi(\delta \vec{r})=\frac{\gamma Z_{P} e}{\sqrt{(\delta x-b)^{2}+\gamma^{2}\left(\delta y-v_{P} t\right)^{2}+\delta^{2} z^{2}}}, \\
\vec{A}(\delta \vec{r})=\frac{\vec{v}_{P}}{c} \Phi(\delta \vec{r}) \equiv(0, \beta \Phi(\delta \vec{r}), 0) .
\end{gathered}
$$

Here the vector with Cartesian components $\left(b, v_{p} t, 0\right)$ specifies the coordinate of relative motion between the centers of mass of the projectile and target and the factor $\delta$ is the one already introduced in Sec. II.

Calling $\Psi(\vec{r}, t)$ the wave function that satisfies the timedependent Schrödinger equation

$$
H \Psi(\vec{r}, t)=\left[H_{0}+V(t)\right] \Psi(\vec{r}, t)=i \hbar \frac{\partial}{\partial t} \Psi(\vec{r}, t)
$$

and expanding this time-dependent solution in the basis of stationary states of the unperturbed Hamiltonian $H_{0}$,

$$
\Psi(\vec{r}, t)=\sum_{\alpha=0}^{\infty} a_{\alpha}(t) \phi_{\alpha}(x, y, z) \exp \left(-\frac{i}{\hbar} \epsilon_{\alpha} t\right),
$$

we easily arrive to a set of coupled differential equations for the amplitudes $a_{\alpha}(t)$, namely

$$
\frac{d a_{n}(t)}{d t}=-\frac{i}{\hbar} \sum_{m}\langle n|V| m\rangle \exp \left(\frac{i}{\hbar}\left(\epsilon_{n}-\epsilon_{m}\right) t\right) a_{m}(t) .
$$

The degeneracies of the one- and two-phonon states are three and six, respectively. In an angular-momentum coupling scheme we understand these as the three projections of $L=1$ for the one-phonon state and the five and one projections of the $L=2$ and $L=0$ components of the double-phonon states. Here, however, we are only interested on the total excitation probabilities of the GDR and the DGDR ignoring anharmonicities and thus we are not necessarily keen to work with this particular coupling scheme. For our present purpose it is most convenient, instead, to use the product basis of harmonic states in Cartesian coordinates. In such a case we identify the global label $\alpha$ of Eq. (3.6) with the trio of numbers $\left(n_{x}, n_{y}, n_{z}\right)$ which gives the number of phonons excited in each of the coordinates $x, y$, and $z$. The reason for this choice is that we can, in good approximation, exclude the possibility of excitation in the direction perpendicular to the reaction plane, being this a motion that is classically forbidden by symmetry considerations. With this in mind we can ignore the quantum label $n_{z}$ and identify the relevant states of our problem with only the pair of numbers $\left(n_{x}, n_{y}\right)$. The effective degeneracy of the one- and two-phonon levels is now two and three, respectively, and we can reduce the space to set-up the coupled-channel problem to just six states, as shown in Fig. 1.

\section{Coupling Scheme}

$\left(n_{x}, n_{y}\right)$

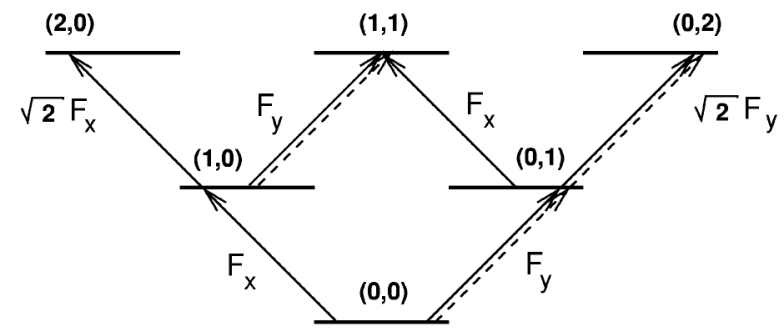

$F_{x}:$ formfactor in space $x$ (real - )

$\mathrm{F}_{\mathrm{y}}:$ formfactor in space y (complex $-+i----$ )

FIG. 1. (Color online) Model space and coupling scheme used in the coupled-channel calculation.

Neglecting the contribution of the $\vec{A} \cdot \vec{A}$ term and expanding the coupling in the neighborhood of the origin to leading order in the coordinates $\vec{r}, \vec{p}$, we obtain

$$
\begin{aligned}
V(\vec{r}, \vec{p}, t)= & V(0,0, t)+G_{1}(t) x+G_{2}(t) y+G_{3}(t) p_{y} \\
& + \text { higher order terms, }
\end{aligned}
$$

where

$$
\begin{gathered}
G_{1}(t)=\frac{\delta Z_{P} Z_{T} e^{2} \gamma b}{\left(b^{2}+\gamma^{2} v_{P}^{2} t^{2}\right)^{3 / 2}}, \\
G_{2}(t)=\frac{\delta Z_{P} Z_{T} e^{2} \gamma^{3} v t}{\left(b^{2}+\gamma^{2} v_{P}^{2} t^{2}\right)^{3 / 2}}, \\
G_{3}(t)=\frac{\delta Z_{P} Z_{T} e^{2} \gamma \beta}{D c\left(b^{2}+\gamma^{2} v_{P}^{2} t^{2}\right)^{1 / 2}} .
\end{gathered}
$$

The zero-order term in the expansion only contributes to the diagonal matrix elements and it is not important in the process. One can now easily take nondiagonal matrix elements of the other terms and derive the relevant couplings, namely a real form factor $F_{x}$ for the excitation of the state $(1,0)$ and a complex formfactor $F_{y}^{R}+i F_{y}^{I}$ for the excitation of the state $(0,1)$,

$$
\begin{gathered}
F_{x}(t)=\sqrt{\frac{\hbar \omega}{2 C}} G_{1}(t), \quad F_{y}^{R}(t)=\sqrt{\frac{\hbar \omega}{2 C}} G_{2}(t), \\
F_{y}^{I}(t)=\hbar \sqrt{\frac{C}{2 \hbar \omega}} G_{3}(t) .
\end{gathered}
$$

As indicated in Fig. 1, all the couplings between the six members of our truncated, harmonic basis of states are simply connected to the form factors for the excitation of the one-phonon states. 

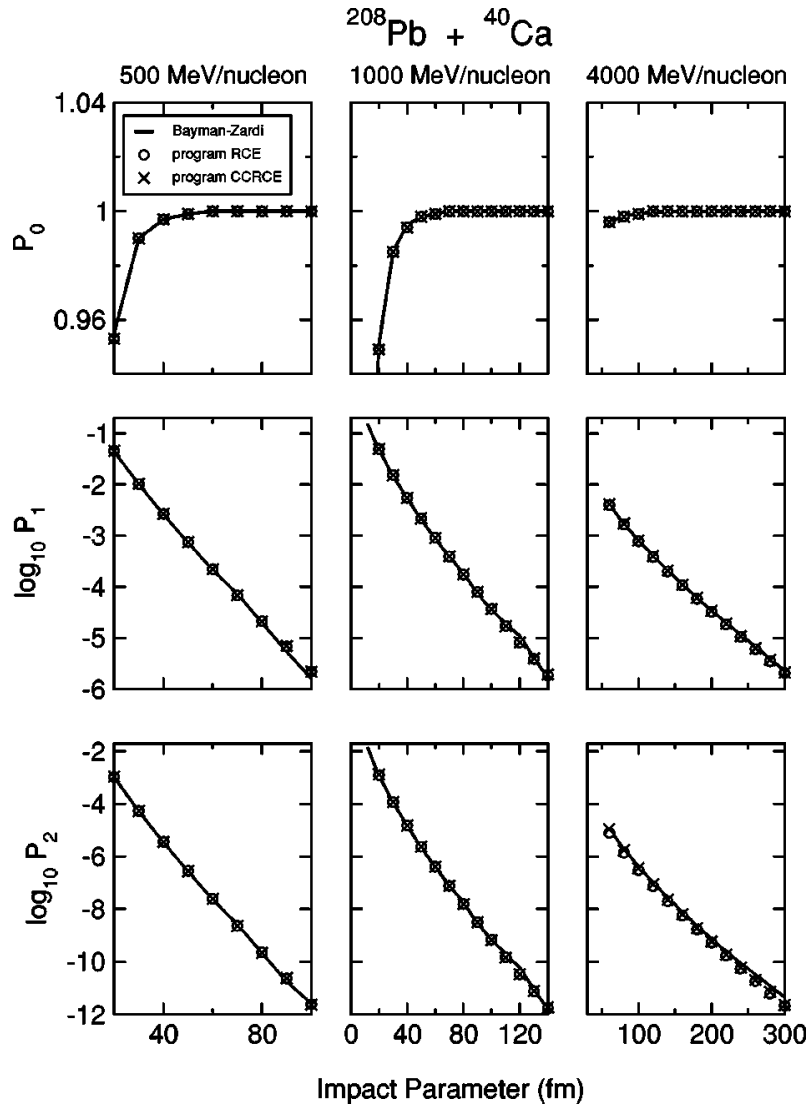

FIG. 2. Probabilities for the excitation of the GDR $\left(P_{1}\right)$ and the double GDR $\left(P_{2}\right)$ in ${ }^{208} \mathrm{~Pb}$ in the reaction ${ }^{40} \mathrm{Ca}+{ }^{208} \mathrm{~Pb}$ at different bombarding energies $(500,1000,4000 \mathrm{MeV}$ per nucleon). The energy of the GDR was assumed to be $11 \mathrm{MeV}$. In the first row the probability $P_{0}$ for remaining in the elastic channel is also shown. The three sets of results give the predictions obtained in the classical model (RCE), in the schematic coupled-channel model (CCRCE), and in the conventional approach (Bayman-Zardi).

A computer code CCRCE has been written (see Appendix B) to construct the excitation probabilities according to this second scheme. We show results and compare with the standard formalism in the following section.

\section{COMPARISON WITH THE STANDARD APPROACH}

We have tested the prescriptions described in the previous sections for the Coulomb excitation of the giant dipole resonance of ${ }^{40} \mathrm{Ca}$ in the reaction ${ }^{208} \mathrm{~Pb}+{ }^{40} \mathrm{Ca}$ at the relativistic bombarding energies of 500, 1000, and $4000 \mathrm{MeV}$ per nucleon. We have considered the probabilities of excitation to the one- and two-phonon states, assuming an arbitrary energy of the GDR of $11.6 \mathrm{MeV}$. Figure 2 displaystogether with the probability of remaining in the elastic channel-the probabilities of exciting the one- and the twophonon states. In the latter case the results include the contribution of both angular momentum components, $L=0$ and $L=2$. The calculation has been performed for a large range of the impact parameters. In the figure we plot the results obtained with the classical formalism of Sec. II (circles) and with the schematic coupled-channel formalism of Sec. III (crosses). Both are compared with the predictions obtained by Bayman and Zardi [18] making use of the standard formalism of Alder and Winther.

The comparison is extremely satisfactory. Although over the considered ranges of impact parameters and bombarding energies the excitation probabilities of the GDR and the DGDR change by many orders of magnitude, all three calculation schemes yield practically the same results. It is in particular quite remarkable to see how the quantal description of the process, involving the construction of the coupling interactions and an elaborate bookkeeping of the probability flow through all the different transitional states, can be so well modelled by the simple elongation of a classical spring.

\section{STUDY OF THE RECOIL}

We said, earlier, that it is customary to set up the coupledchannel formalism in the system of reference in which the dipole mode (the spring in our case) is at rest. This is not correct, since we are not here talking of a classical pointdipole made by equal positive and negative charges separated by a certain distance $d$. The nucleon world is not symmetric in charge and the counterpart to the positively charged protons are the chargeless neutrons. As such, we cannot ignore that the leading contribution in our multipole expansion of the external field is not given by the dipole coupling but by the monopole term. In other words, the nucleus whose GDR we want to investigate will be pushed away by the projectile with a repulsive force $Z_{P} Z_{T} e^{2} / R^{2}$ even before the dipole coupling starts to act.

There has been some effort devoted to estimate the consequences of this lack of inertiality in the reference system $[19,20]$. We would like here to do it in our own way, as we think that the working frame developed in Sec. II is particularly suitable to provide an intuitive measure of how things do happen.

The starting point is to realize that we need not invoke the dipole excitation couplings to solve our problem. If we are willing to integrate separately the coordinates of the center of charge and that of the neutral matter the problem is perfectly defined. We need to integrate the equations of motion for the protons (that feel the external field and are "slowed down" by the spring that links them to the neutrons). And, also, for the neutral matter that is forced to follow the protons by the same agent and thus are "dragged" around by their motion.

It is almost easier to implement this idea in practice than to explain it in detail. Let us just say that a program quite analogous to the RCE shown in Appendix A (and not much longer in lines) allows us to calculate the trajectories of the center of charge (protons) and of the neutral matter (neutrons). Of course, the Lorentz force only appears in the equations of motion for the charged component while action and reaction to the Hooks' force appear in either set. This program is called RCE2C.

Eliminating the variable time from the numerical solutions we can plot the projection of the resulting motion in the reaction plane. In the top frame of Fig. 3 we see the separate trajectories for the protons and neutrons that clearly exhibit 

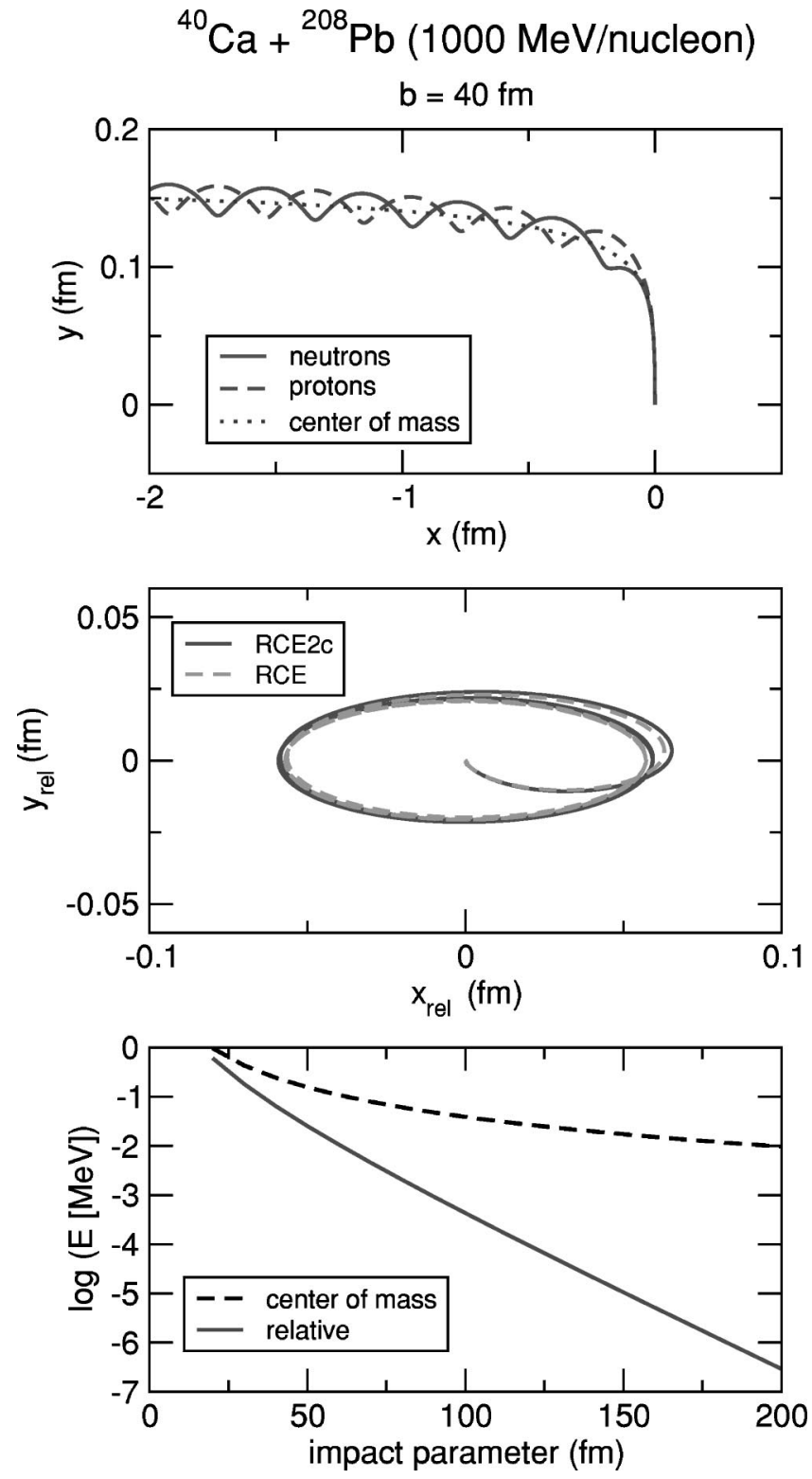

FIG. 3. Upper frame: trajectory of the proton center of mass (solid line), of the neutron center of mass (dashed line) and the total center of mass (dotted line) in the reaction ${ }^{40} \mathrm{Ca}+{ }^{208} \mathrm{~Pb}$ at an energy of $1000 \mathrm{MeV} /$ nucleon and an impact parameter $b=40 \mathrm{fm}$. Middle frame: trajectory of the relative motion between protons and neutrons, projected in the reaction plane $\left(x_{\text {rel }}, y_{\text {rel }}\right)$. Lower frame: energy of the center of mass (dashed line) and energy of the relative coordinate (full line) as a function of the impact parameter for the same reaction.

an oscillatory behavior around the total center of mass indicated by the dotted line. We want to stress that it does not need to be always so. The strength of the restoring forces of the spring and the time scale of the driving electromagnetic forces are fundamental to determine what kind of motion will actually ensue.

The displacement of the center of mass is rather small during the collision time. This makes it reasonable to expect a minor effect on the magnitude of the couplings as a con-

sequence of their radial dependence. This is certainly so for the long-range monopole Coulomb field, but could become more critical for short-range coupling such as is the case with nuclear form factors.

The center frame in Fig. 3 compares the results for the trajectories of relative motion obtained with the program $\mathrm{RCE}$ of Sec. II and the new RCE2C. It is seen that the overall (accelerated) displacement of the center of mass has no appreciable consequences on the oscillatory motion performed classically by the GDR. Since it is the amplitude of this asymptotic elliptical motion that essentially determines the probabilities of excitation of the one- and two-phonon states of the giant resonance the results of both codes essentially coincide.

One could think that this may be the case only for small impact parameters. In fact, for $b=40 \mathrm{fm}$ (the impact parameter used to generate the top two frames in the figure) the energy invested in the recoil of the target and the intrinsic energy are of the same order of magnitude. As the impact parameter increases, however, one expects that the recoil energy will reduce gradually, as it follows from the slow $1 / r$-dependence of the monopole Coulomb field. The excitation of the dipole mode should, on the other hand, decrease much faster, in comparison, due to two reasons. Namely, the more pronounced radial drop of the coupling and an adiabatic cut-off factor that is quite effective for the high excitation energies characteristics of the GDR. Notice that the presence of this factor affects the energy transfer to the intrinsic system but not the energy invested in the recoil. In short, the relationship between the energy acquired by the intrinsic system and that spent in the recoil should become completely lopsided in favor of the latter as the larger impact parameters are probed. This argument is dramatically confirmed in the lower frame of the figure, where the logarithmic vertical scale indicates that for impact parameters above $\approx 100 \mathrm{fm}$ practically all the energy loss in the beam is invested in making the target recoil.

The absolute values of the energies involved for large impact parameters are, however, quite small numbers and the inertial forces that enter into play should be still smaller than the ones acting for our example at $b=40 \mathrm{fm}$. It should thus come as no surprise to see the results displayed in Fig. 4, where it is shown that the agreement of the calculations done with RCE2C with those of Bayman and Zardi is equally good as the one previously reported in Sec. IV.

\section{SUMMARY AND CONCLUSIONS}

In this contribution we have explored the possibility of approaching the relativistic Coulomb excitation problem avoiding the inherent complexity of the standard formulation of Alder and Winther. For this we relied only on much of what has been learned applying semiclassical techniques and approximate quantization procedures in the analysis of heavy ion reactions. The results we get are extremely good when compared with those obtained with the conventional formalism. We want to stress that we have here aimed to compare directly with what are called the exact results, thus bypassing different schemes introduced in the literature to approach 

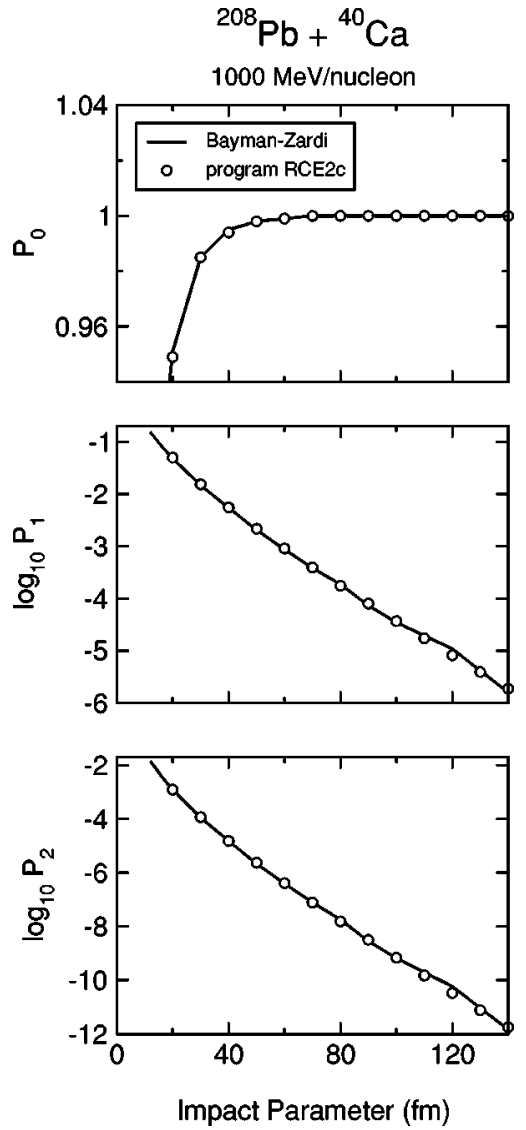

FIG. 4. Probabilities for the excitation of the GDR $\left(P_{1}\right)$ and the double GDR $\left(P_{2}\right)$ in ${ }^{208} \mathrm{~Pb}$ in the reaction ${ }^{40} \mathrm{Ca}+{ }^{208} \mathrm{~Pb}$ at bombarding energy of $1000 \mathrm{MeV}$ per nucleon. In the upper the probability $P_{0}$ for remaining in the elastic channel is also shown. The points give the predictions obtained by the formalism in which the target nucleus is allowed to recoil (RCE2C) compared with the results by Bayman and Zardi.

that elusive goal (Born approximations, long-wavelength approximations). Of course, the previous statement about agreement should be qualified with a range of bombarding energies. What we can say is that our alternative works at least up to $\approx 5 \mathrm{GeV} /$ nucleon; the emphasis being there to stress that, at the moment, we cannot test precisely its actual range of validity. Could be more. The quoted value covers generously, in any case, the beam energies that are used in current investigations of the DGDR.

Coupled-channel calculations performed in a space truncated at the two-phonon level develop a problem for very small impact parameters. It occurs when, along the way, the probability flow needs to exceed that self-imposed ceiling. This source of errors is, as a matter of fact, something that is common to both our formulation and the standard one. The trouble is potentially serious because the asymptotic probabilities, at the end of the trajectories, are such that they almost always satisfy $P_{2} \ll P_{1} \ll 1$. This may create a false sense of security and lead the analyst to believe that the results are compatible with the assumptions made when truncating the model space. One has to check carefully that at all times the flow of probability remains within the restricted number of channels. (We have of course done this and also have written a variation of the program given in Appendix B that incorporates a larger space to check the convergency of the results.) It is interesting, incidentally, to mention that while the truncation of the space is crucial to limit the validity of the coupled-channel approach it does not affect in a similar way the simplest of the calculation schemes we have proposed (namely, the program RCE of Appendix A; quite simply, there is no truncation in this approach). Indeed, it was shown in a study of the excitation of vibrational modes in the chaotic regime [21] how the nuclear surface is capable-by stretching what is essentially only a bit more- to yield average results that can be accurately converted into probabilities for the population of the one- and two-phonon levels.

We have included in Appendices A and B the listings of two (conceptually very different) programs to calculate the relativistic Coulomb excitation of giant dipole modes. This is unusual insofar as appendices are normally used to relegate all the difficult formulas that the authors wish to weed out from the text. Here we wanted to use them to show something simple. We think that it may be a source of pleasure for those that have struggled to implement the Alder-Winther formalism to see that nearly identical results can be obtained by numerical procedures that are that straightforward. Also, because it may be useful for those planning experiments and who cannot use any other alternative to have at their disposal a simple way to estimate the probabilities of excitation of the GDR and the DGDR for different nuclei and different bombarding conditions. The content of the appendices will allow the reader to check all the points presented in Fig. 2. Please note, however, that in order to fit the codes into the format of this contribution the listings have been compacted into a bare minimum. It is trivial to incorporate a loop over the impact parameters, calculate cross sections, etc.

\section{ACKNOWLEDGMENTS}

We would like to thank Ben Bayman and Franco Zardi for many discussions and suggestions.

\section{APPENDIX A}

Simple program RCE to calculate the probabilities of excitation for the first three steps of the harmonic ladder of the Giant Dipole mode according to the content of Sec. II. The code is self-contained, except for a single line that calls an integration routine, indicated by the arrow. In this case we use the routine D02BAF of the NAG library [22] but can be replaced by any other available to the reader. 


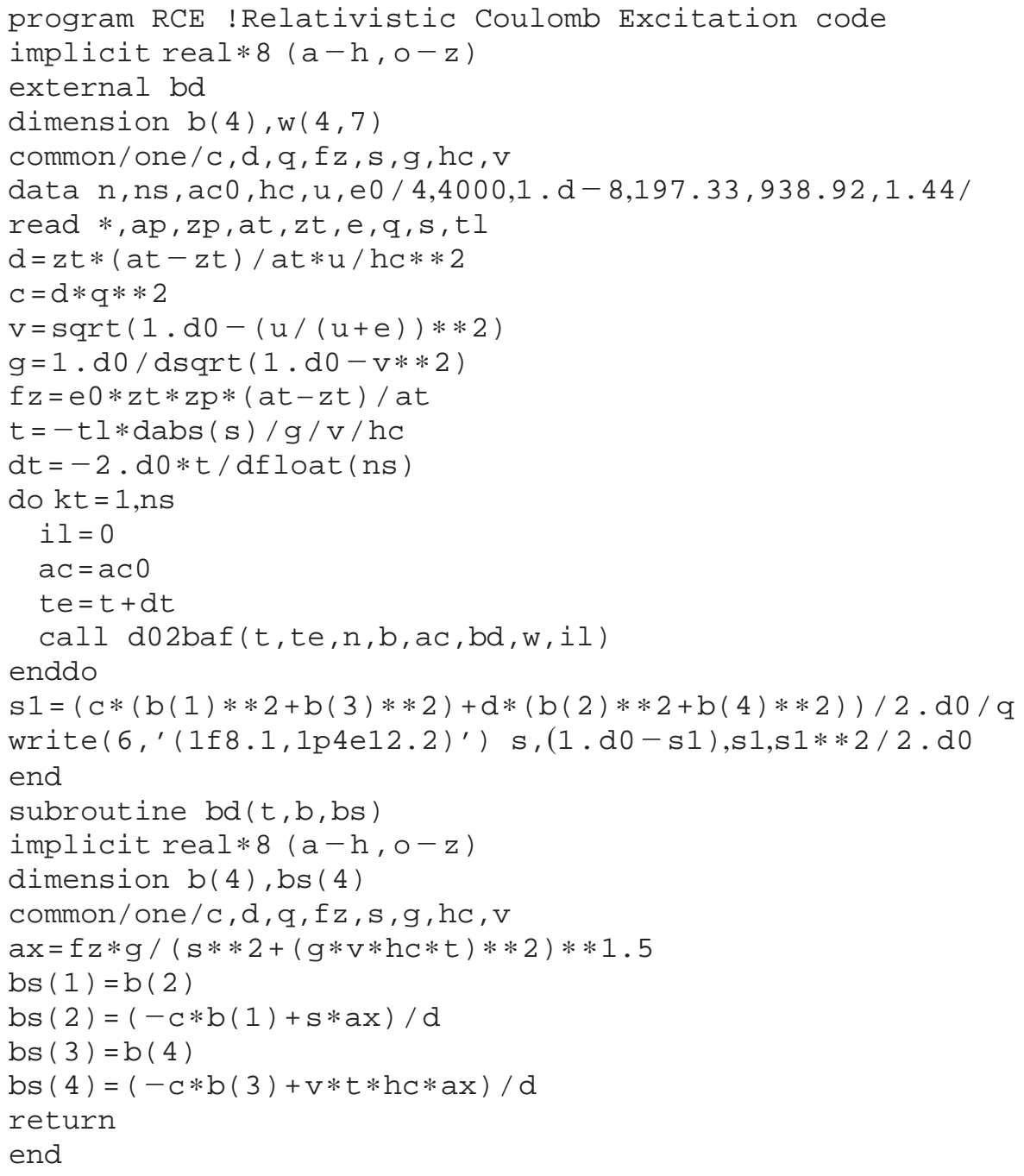

\section{APPENDIX B}

Simple program CCRCE to calculate the probabilities of excitation for the first three steps of the harmonic ladder of the giant dipole mode according to the content of Sec. III. The code is complete and self-contained, except for one call to the integration routine D02BAF of the NAG library, just as it was the case in RCE (see Appendix A).

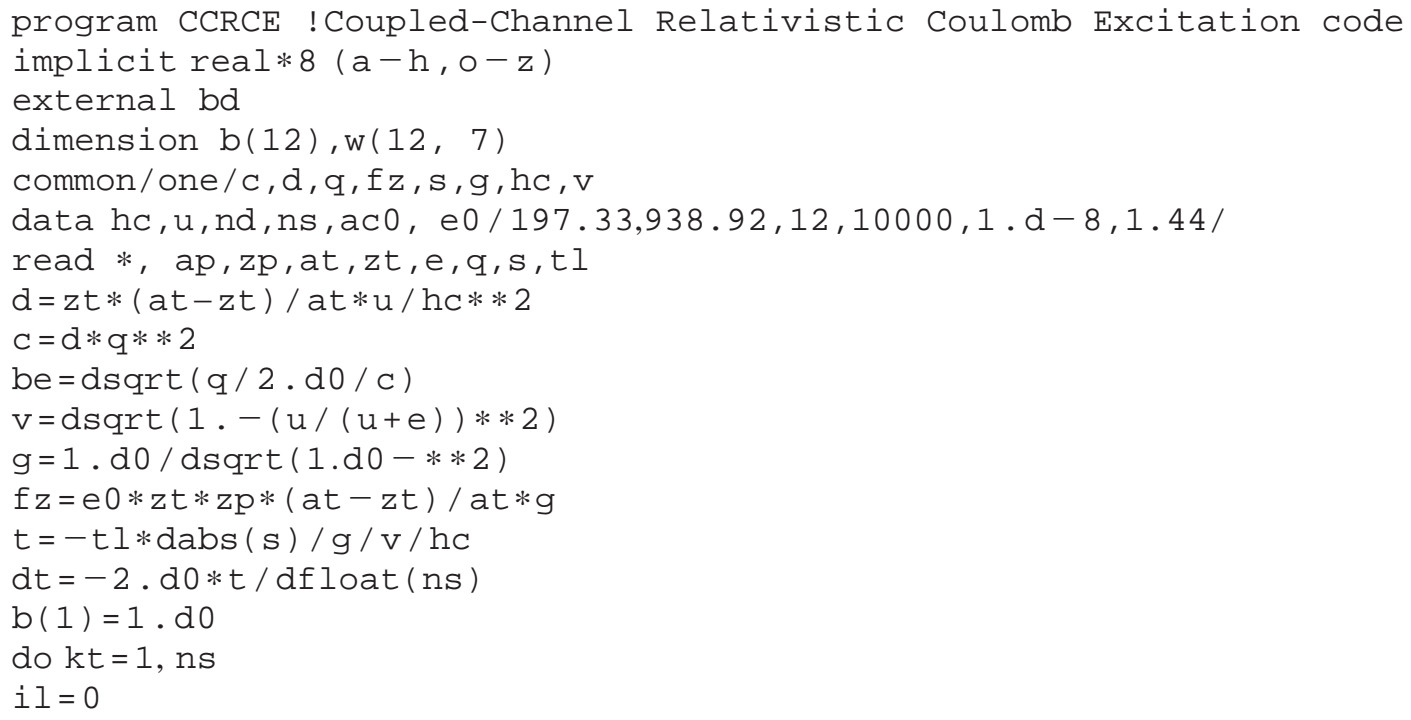




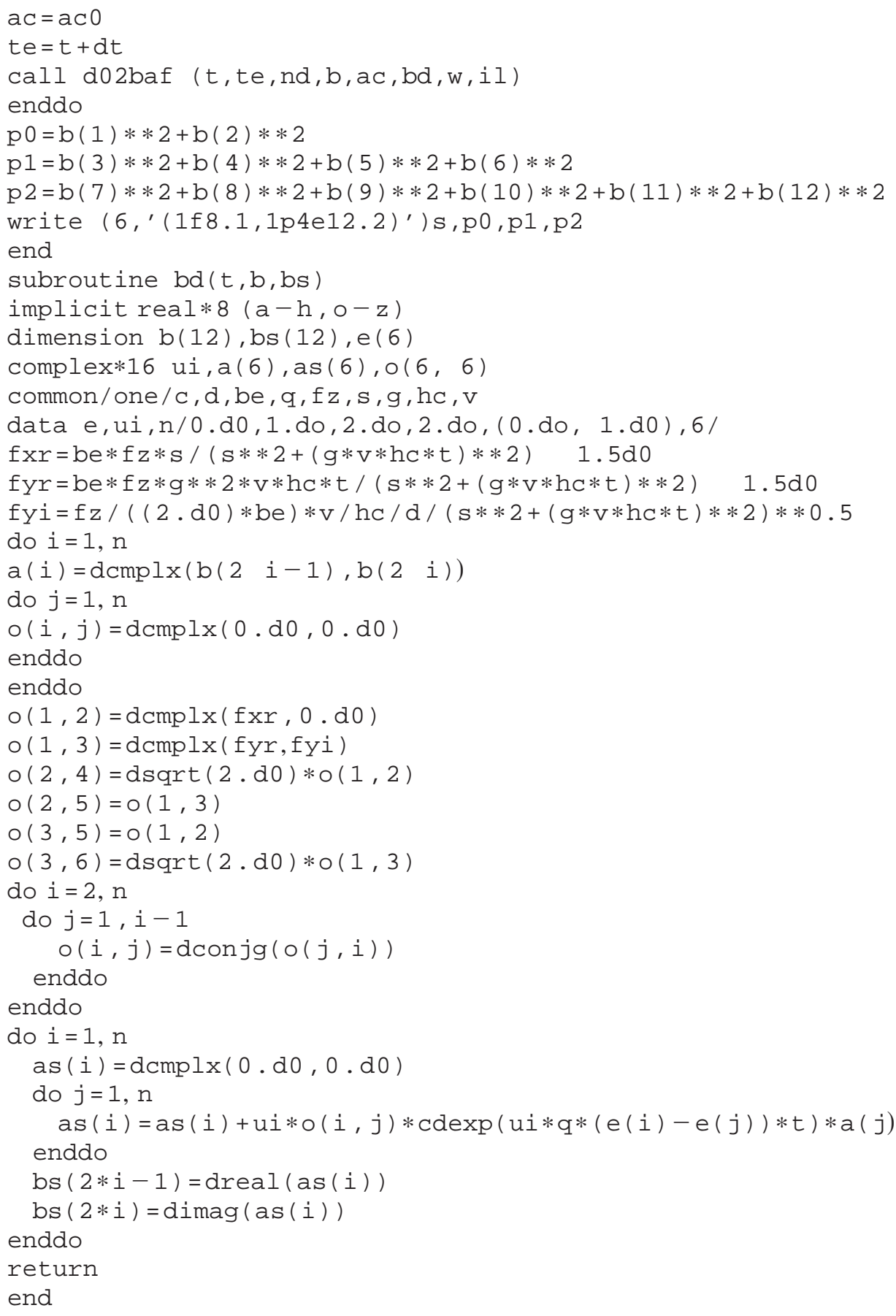

[1] K. Alder and A. Winther, Electromagnetic Excitation (NorthHolland, Amsterdam, 1975).

[2] L.C. Biedenharn and P.J. Brussaard, Coulomb Excitation (Clarendon, Oxford, 1965).

[3] C.A. Bertulani and G. Baur, Phys. Rep. 163, 299 (1988).

[4] V.Yu. Ponomarev, E. Vigezzi, P.F. Bortignon, R.A. Broglia, G. Colò, G. Lazzari, V.V. Voronov, and G. Baur, Phys. Rev. Lett. 72, 1168 (1994).

[5] C.A. Bertulani, F. Canto, M.S. Hussein, and A.F.R. de Toledo
Piza, Phys. Rev. C 53, 334 (1996).

[6] E.G. Lanza, M.V. Andres, F. Catara, Ph. Chomaz, and C. Volpe, Nucl. Phys. A613, 445 (1997).

[7] P.F. Bortignon and C.H. Dasso, Phys. Rev. C 56, 574 (1997).

[8] E.G. Lanza, M.V. Andres, F. Catara, Ph. Chomaz, and C. Volpe, Nucl. Phys. A636, 452 (1998).

[9] B.F. Bayman and F. Zardi, Phys. Rev. C 59, 2189 (1999).

[10] C.A. Bertulani and V.Yu. Ponomarev, Phys. Rep. 321, 139 (1999). 
[11] B.V. Carlson, L.F. Canto, S. Cruz-Barrios, M.S. Hussein, and A.F. R. de Toledo Piza, Phys. Rev. C 59, 2689 (1999).

[12] B.V. Carlson, M.S. Hussein, A.F. R. de Toledo Piza, and L.F. Canto, Phys. Rev. C 60, 014604 (1999).

[13] M.S. Hussein, A.F.R. de Toledo Piza, and O.K. Vorov, Phys. Rev. C 59, R1242 (1999).

[14] V.Yu. Ponomarev, P.F. Bortignon, R.A. Broglia, and V.V. Voronov, Nucl. Phys. A687, 170c (2001).

[15] D.T. de Paula, T. Aumann, L.F. Canto, B.V. Carlson, H. Emling, and M.S. Hussein, Phys. Rev. C 64, 064605 (2001).

[16] A. Winther and K. Alder, Nucl. Phys. A319, 518 (1979).
[17] J.D. Jackson, Classical Electrodynamics (Wiley, New York, 1975).

[18] B.F. Bayman and F. Zardi, Phys. Rev. C 68014905 (2003).

[19] C.A. Bertulani, A.E. Stuchbery, T.J. Mertzimekis, and A.D. Davie, Phys. Rev. C 68, 044609 (2003).

[20] B.F. Bayman (private communication).

[21] C.H. Dasso, M. Gallardo, and M. Saraceno, Nucl. Phys. A587, 339 (1995), and (to be published).

[22] NAG Fortran Library (The Numerical Algorithms Group Ltd., Oxford). 